\title{
A Continuum Model of Gas Flows with Localized Density Variations
}

\author{
S. Kokou Dadzie, Jason M. Reese* and Colin R. McInnes \\ Department of Mechanical Engineering, University of Strathclyde, \\ Glasgow G1 $1 X J, U K$
}

\begin{abstract}
We discuss the kinetic representation of gases and the derivation of macroscopic equations governing the thermomechanical behavior of a dilute gas viewed at the macroscopic level as a continuous medium. We introduce an approach to kinetic theory where spatial distributions of the molecules are incorporated through a meanfree-volume argument. The new kinetic equation derived contains an extra term involving the evolution of this volume, which we attribute to changes in the thermodynamic properties of the medium. Our kinetic equation leads to a macroscopic set of continuum equations in which the gradients of thermodynamic properties, in particular density gradients, impact on diffusive fluxes. New transport terms bearing both convective and diffusive natures arise and are interpreted as purely macroscopic expansion or compression. Our new model is useful for describing gas flows that display non-local-thermodynamic-equilibrium (rarefied gas flows), flows with relatively large variations of macroscopic properties, and/or highly compressible fluid flows.
\end{abstract}

Key words: gas kinetic theory, Boltzmann equation, compressible fluids and flows, Navier-Stokes equations, rarefied gas dynamics, constitutive relations

\section{Introduction}

In gas dynamics, the Boltzmann kinetic equation is generally accepted as describing the evolution of the dilute gaseous molecule distribution function. This equation is presumed to be valid for any dilute gas flow, whatever the Knudsen number (which is defined as the ratio of the molecular mean free

* Corresponding author.

Email address: jason.reese@strath.ac.uk (Jason M. Reese). 
path to a typical macroscopic length scale of the flow) [1]. The Boltzmann kinetic equation has received a number of derivations under specific assumptions which all aim to prove its physical and mathematical rigour [2]. It is also widely accepted that continuum fluid mechanical models, and associated classical thermodynamic models, in particular equations of state, can be obtained from the statistical kinetic formulations [3]. For example, it is well-known that the three classical fluid dynamic equations, the Navier-Stokes equations, may be derived from the Boltzmann kinetic equation [4]. These fluid mechanical equations have amply demonstrated their capability in handling typical flows of small Knudsen number, or flows with relatively small variations of their macroscopic properties.

Describing flows beyond the broad range of applicability of the Navier-Stokes model remains, however, a critical area of active investigation. These flows range from the high speed (e.g. shock waves, kinetic boundary layers, reentry problems) to the micro- and nano-scale. Heat transfer processes in a highly rarefied gas, in particular at high temperature gradients [5-7], and some thermally-driven flows are also not well understood [8-10]. Meanwhile, various continuum models based on approximate solutions to the Boltzmann equation, and which are expected to cover flows beyond the Navier-Stokes level, are the subject of different controversies and still under investigation $[9,11-15]$.

In this paper we discuss the kinetic description of dilute gases, and its link to continuum hydrodynamic models for gas flows. We revisit the statistical microscopic representations of a dilute gas where the medium is still measurable by macroscopic quantities, i.e. mean flow velocity and thermodynamic properties (density, temperature, pressure). We introduce a kinetic representation that includes the spatial configuration of the molecular ensemble via the free spaces between the molecules. The fundamental change introduced in our kinetic statement is that, while a gas is composed of molecules rapidly moving and interacting, the free volume around each gaseous molecule should be accounted for in a complete microscopic representation. This statement allows us:

(1) to maintain in the statistical microscopic description a proper account of the collective nature of the medium constituted by the molecules;

(2) to consider the domain (with its boundaries) occupied by an ensemble of molecules locally as an independent variable that is free from any reference frame, in accordance with classical thermodynamics.

In the first section of this paper we review some of the mathematical tools presently used in statistical mechanics and kinetic theory to derive non-equilibrium gas equations; in particular, the probability density and its link to fluid massdensity. Subsequent sections are devoted to the formulation of an alternative 
kinetic description and consequent hydrodynamic models. In the final section we present some qualitative predictions of the new model compared with the traditional Boltzmann model and continuum fluid equations.

It is important to note that, while the discussion presented in this paper may impact on extended versions of the Boltzmann kinetic equation, we do not directly consider here the broad range of modified versions of the Boltzmann kinetic equation used in other fields such as dense media, media comprising heavy and large molecules, reacting and ionized gases, molecules with rotational and vibrational energies or molecules with internal structures, mixtures etc. We are concerned only with the monatomic elementary gas kinetic equations, in which molecules are viewed as point masses.

This paper describes the full development of a model the authors have been considering over recent years [16].

\section{Preliminaries}

\subsection{Classical kinetic equations vs. molecular spatial distributions}

The physical space is referenced with a fixed inertial frame $\left(X_{1}, X_{2}, X_{3}\right)$, in which exists a gas. We denote a differential element in the position sub-phase space, $d_{X}=d_{X_{1}} d_{X_{2}} d_{X_{3}}$, and a differential element in the velocity sub-phase space, $d_{\xi}=d_{\xi_{1}} d_{\xi_{2}} d_{\xi_{3}}$. Let us define the following two probability densities:

(A) A probability density function $f_{A}(t, X, \xi)$ such that $f_{A}(t, X, \xi) d_{X} d_{\xi}$ represents the probable number of molecules that, at time $t$, have their positions located within $X \pm d_{X}$ and their velocities within the element $\xi \pm d_{\xi}$.

(B) A probability density function $f_{B}(t, X, \xi)$ for an arbitrary single gas molecule such that $f_{B}(t, X, \xi) d_{X} d_{\xi}$ represents the probability that, at time $t$, the velocity of this single molecule is within the element $\xi \pm d_{\xi}$ and the position of this single molecule is within $X \pm d_{X}$.

The above two probability density functions are evidently two different concepts. In particular, (A) gives a number of molecules, while (B) does not. We use the term "mass-density" for the physical "density" of a medium as it is conceived in classical continuum mechanics as a thermodynamic property, and it is viewed as an amount of mass divided by the volume in which is spread this mass. This volume is, in reality, made up of empty spaces and real volumes of the molecular objects. The volume, in which is spread the mass, is itself a thermodynamic variable in classical equilibrium thermodynamics. Therefore the mass-density, and its associated specific volume, have at first 
sight no predefined assignment to the mathematical measures or probability density functions. Nevertheless, a rational derivation of fluid kinetic equations evidently proceeds through a proper handling of this quantity with mathematical tools.

A derivation of the Boltzmann kinetic equation in a monatomic dilute gas may start with the Liouville equation for the one-molecule distribution function, as defined in (B), see also [17]. The Liouville equation is written

$$
\frac{\partial f_{B}}{\partial t}+(\xi \cdot \nabla) f_{B}+\left(F_{t o t} \cdot \nabla_{\xi}\right) f_{B}=0
$$

where $\nabla=\left(\partial / \partial X_{1}, \partial / \partial X_{2}, \partial / \partial X_{3}\right)$ is the traditional spatial gradient operator and $\nabla_{\xi}$ denotes the similar operator in the velocity space, i.e., $\nabla_{\xi}=$ $\left(\partial / \partial \xi_{1}, \partial / \partial \xi_{2}, \partial / \partial \xi_{3}\right)$. In equation (1), the third term on the left hand side corresponds to the total force exerted on a given arbitrary molecule. This force encompasses both external actions and the potential forces exerted by the other surrounding molecules. The route to a gas kinetic equation is concerned with modelling the force term. Instead of maintaining the continuous action of the intermolecular forces, this force component is replaced by discontinuous changes that occur instantly onto the momentum of the single molecule [17]. The resulting equation is the Boltzmann equation in the one-particle phase space, written when ignoring external forces as

$$
\frac{\partial f_{B}}{\partial t}+(\xi \cdot \nabla) f_{B}=I\left(f_{B}, f_{B}\right)
$$

The term on the right hand side that arose from the intermolecular forces is the collision integral, restricted by the assumption that molecules are uncorrelated in both the position and velocity spaces. For hard-sphere molecules, this is written,

$$
I(f, f)=\int\left[f\left(t, X, \xi^{*}\right) f\left(t, X, \xi_{1}^{*}\right)-f(t, X, \xi) f\left(t, X, \xi_{1}\right)\right] \xi_{r} b d_{b} d_{\epsilon} d_{\xi_{1}},
$$

where $\xi$ and $\xi_{1}$ refer to post-collision velocities of the interacting molecules, $\xi^{*}$ and $\xi_{1}^{*}$ refer to pre-collision velocities, $\xi_{r}=\left|\xi-\xi_{1}\right|$ is the two colliding molecules' relative velocity, $\epsilon$ is the azimuthal impact angle, $b$ is the distance of closest approach of the undisturbed trajectories in the centre-of-mass frame of reference. We recall that this collision integral is based on the elementary dynamic laws of a collision between two point-mass molecules, only exchanges of momentum and energy are involved, and that this collision integral does not describe any spatial configuration changes during the collisions.

Let us define the following quantity:

$$
B_{n}(t, X)=\int f_{B}(t, X, \xi) d_{\xi},
$$


which is another probability density function in the position sub-space. According to the definition of the distribution function $f_{B}, B_{n}(t, X) d_{X}$ represents the probability of finding the single molecule in the vicinity of position $X$, regardless of its velocity. In kinetic theory, $B_{n}(t, X)$ is conventionally associated with the mass-density of the medium through the following assertion [18]: consider a fixed number $N$ as the total number of gas molecules; assume

$$
f_{A}=N f_{B}
$$

and then interpret $\int f_{A} d_{\xi}$ as an average number of molecules per unit gas volume; hence $B_{n}(t, X)$ (or $N B_{n}(t, X)$ ). An elementary volume of gas is also then represented by $d_{X}$, and the distribution function $f_{A}$ follows equation (2) because $N$ is just a constant.

However, this assertion, and equation (5), presupposes that the summation over the one-particle distribution functions corresponding to each individual molecule gives the average number of molecules around a given position. This implies that each molecule is statistically independent (this is without referring to the collision integral), which means the true collective nature of the molecules constituting the medium, and the real spatial configurations of the molecules, are disregarded. For example, the position of a given molecule relative to another is ignored. Furthermore, an elementary volume of gas represented by $d_{X}$ within this definition is a frame-dependent quantity, in contrast with thermodynamic properties.

Generally, an approximation such as equation (5) compromises a good description of the mass-density in a non-equilibrium gas. Indeed, it is important to note that in a non-equilibrium gas the thermodynamic properties of the gas medium are defined locally. It is a localized number of molecules that defines the macroscopic mass-density, and this varies in time and position. Some published articles containing derivations of the Boltzmann kinetic equation explicitly present the mass-density of the gas as a constant, or the medium as spatially uniform $[19,20]$. The mass-density of the medium is defined as a normalization factor $N / V$ in front of a distribution function, where $N$ is the total number of molecules in a fixed volume $V$ of a container [20,21].

Derivations of the Boltzmann equation from the Liouville equation are also frequently based on an asymptotic limit analysis that involves $N$ tending to infinity. However, for the distribution function described in (A), when the number of molecules tends to infinity then the distribution function $f_{A}$ also tends to infinity (the number of molecules per unit of physical volume becomes infinite). Then a rescaling, such as equation (5), is usually required [22]. Therefore, even with equation (5), the definition of $B_{n}$ in equation (4) is difficult to classify as a physical mass-density of the medium being a thermodynamic variable. 
Another common route to the Boltzmann kinetic equation is to start with the distribution function $f_{A}$ directly, so that equation (4), written with $f_{A}$ instead, reads as an average number of molecules per unit volume, which is then interpreted as the mass-density of the medium. In this derivation, it is simply assumed that "the variation of a number of molecules in a cell defined by $\left(\xi \pm d_{\xi}\right) \otimes\left(X \pm d_{X}\right)$ is due to collision between molecules" [4], and that collision itself is an operation occurring only in the velocity sub-space. Then,

$$
\frac{\partial f_{A}}{\partial t}+(\xi \cdot \nabla) f_{A}=I\left(f_{A}, f_{A}\right)
$$

There are some incompatibilities inherent in this formulation. Considering a position $X$ in the gas, there is not a given single molecule but a collection of molecules associated with that point, and in a similar manner the velocity (some references use the term "molecules of a kind" [4,23]). Accordingly, collisions can be regarded as interactions between two groups of molecules. This contrasts with the usual description of the dynamics of a collision as an interaction between two individual molecules coming from two different positions. The concept of many molecules sitting at the same position at the same time can be regarded as problematic in equation (6). In any case, an elementary volume of a gas, that is, the differential element $d_{X}$, is still linked to the frame $\left(X_{1}, X_{2}, X_{3}\right)$, in contrast to the classical thermodynamic concept of the volume of gas. Note that if a gas is flowing while in non-equilibrium, then the domain occupied may be both variable and moving, while the reference frame attached to $X$ will always be fixed.

\subsection{Equilibrium distribution and the Boltzmann kinetic equation}

According to equation (2), equilibrium holds if and only if the distribution corresponds to the Maxwell-Boltzmann distribution given by (and disregarding external force fields such as gravity),

$$
F_{0}\left(V_{m}\right)=\left(\frac{M}{2 \pi k T_{B}}\right)^{\frac{3}{2}} \exp \left[-\frac{M}{2 k T_{B}} V_{m}^{2}\right],
$$

where $V_{m}$ is the molecular random velocity, $T_{B}$ is the kinetic temperature, $M$ is the gaseous molecular mass; and $k$ the Boltzmann constant. Note that neither the mass-density of the medium nor any pressure distributions are involved in this distribution function; only the kinetic energy of the molecules, which describes the kinetic temperature, is involved. In particular, the equilibrium is defined by the molecular kinetic energy fluctuating around a constant value, which defines the constant temperature. In spite of its widespread use in nonequilibrium gas dynamics, it is still not completely clear whether such a distribution represents a complete thermodynamic equilibrium of a gas [24,25]; 
we recall that the Boltzmann H-theorem, associated with the derivation of the Maxwell-Boltzmann distribution as the only equilibrium distribution, is strictly derived only if it is admitted first that the medium is spatially uniform, and that bounding wall effects are neglected $[4,18]$.

If the spatial distribution of the molecules is considered, a complete equilibrium distribution takes the form [26]

$$
F\left(V_{m}, \varepsilon_{i}\right)=A \exp \left[-\frac{\varepsilon_{i}}{k T_{B}}\right] \exp \left[-\frac{M}{2 k T_{B}} V_{m}^{2}\right],
$$

where $\varepsilon_{i}$ expresses the potential energy of a given molecule due to the presence of other surrounding molecules, and $A$ is a normalization factor.

Dilute gas dynamics is founded on the assumption that molecules move mostly uniformly, without any change in their momentum and also without any communication with other molecules. Communication only takes place when two molecules move very close to each other, which is the so-called "collision". It is argued that long range forces are not important in a very dilute medium where short-range interactions dominate molecular momentum exchanges. However, this neglects systematically the true collective or cohesive nature of the molecule ensemble, and the description of their real spatial distributions is disregarded (as is seen by comparing equation 7 with equation 8 ). The spatial distributions of the molecules, founded on molecular separation distances, control the evolution of macroscopic thermodynamic variables, such as the gas mass-density, specific volume or pressure.

Characteristics of the traditional Boltzmann equation impose a certain asymmetry between the changes in positions and changes in velocities that is observed in the structure of the equation: spatial variations of the distribution function are independent of interactions between molecules that are contained in the collision integral. A correction of this asymmetry can be based on the concept of non-local and non-instant collisions [3]. However, this asymmetry may also be corrected by supplementing the Boltzmann description with a proper description of spatial variations of the molecules. This is the type of model we investigate in the remainder of this paper.

\section{An alternative kinetic model in continuous media}

\subsection{Formulation}

In the previous section, our main criticism of existing kinetic models was the representation of the mass-density of the medium and the spatial distribu- 
tion of the molecules. In this section, we present a kinetic model of a dilute gas that includes a mass-density representation in the microscopic schema as a property that involves many molecules. The conceptual frame is the following: consider a molecule that belongs to a gas medium; the medium will be measured by macroscopical continuum field variables, so each gaseous molecule should not be described as a single moving molecule at the microscopic level but should be taken together with its surrounding molecules (i.e. together with its environment).

Considering an arbitrary molecule, we define the following probability distribution function:

$f(t, X, \xi, v)$ is such that $f(t, X, \xi, v) d_{X} d_{\xi} d_{v}$ is the probability of an arbitrary single molecule to be, at a given time $t$, located in the vicinity of position $X$ with its velocity in the vicinity of velocity $\xi$, while the configuration of its surrounding molecules at that time is readable with a microscopic parameter whose measurable value is around $v$.

Variable $v$ takes a positive value so that $v$ tending to zero represents packed gaseous molecules with no separation distances, and $v$ tending to infinity represents an isolated molecule. A dilute gas properly lies between these two limiting cases. This new variable bears information about other molecules, and the cohesive nature of the medium, and therefore completes the one-molecule description.

This new variable is independent of the position and velocity variables of a given molecule; it will not be important if continuum field variables based on collections of molecules, such as mass-density and pressure, are not considered (i.e. if we only wish to describe a single moving molecule). More precisely, if $d$ is on average the distance between a target single molecule and its surrounding molecules, then the geometrical variable $v$ may be given a handleable value of the volume of the sphere of radius $(d / 2)$, i.e $v=(4 \pi / 3)(d / 2)^{3}$.

A total variation in time of the new one-molecule distribution function is given by,

$$
\frac{\delta f}{\delta t}=\frac{\partial f}{\partial t}+\left(\frac{\delta X}{\delta t} \cdot \nabla\right) f+\left(\frac{\delta \xi}{\delta t} \cdot \nabla_{\xi}\right) f+\frac{\delta v}{\delta t} \frac{\partial f}{\partial v}
$$

where $\delta / \delta t$ denotes the total time derivative following microscopic motions. The rate of change of position with time is the velocity of the molecule, so $\delta X / \delta t=\xi$. The rate of change of momentum with time is the sum of forces exerted on the molecule, so $\delta \xi / \delta t=F_{\text {ext }}+F_{\text {int }}$, where $F_{\text {ext }}$ denotes external forces such as gravity, $F_{\text {int }}$ denotes internal forces due to other molecules (per unit mass). The last term in equation (9) results from the local change of $v$, i.e. the change in the configuration of the molecular ensemble due to changes in the properties of the medium. We will therefore denote $W=\delta v / \delta t$, and assume this quantity depends on the macroscopic properties of the medium. 
We admit that the probability associated to $f$ is conserved in the generalized phase space $(X, \xi, v)$ and we can therefore write equation (9) as,

$$
\frac{\partial f}{\partial t}+(\xi \cdot \nabla) f+\left(F_{e x t} \cdot \nabla_{\xi}+F_{i n t} \cdot \nabla_{\xi}\right) f+W \frac{\partial f}{\partial v}=0
$$

There are, in equation (10), two main terms to deal with: the change in momentum of the target molecule, and the change in the configuration of its surroundings. These two terms can be modelled separately as, in the statistical description, position and momentum are independent random variables. The variation of $f$ due to the stream motion of the target molecule in space within equation (10) differs from the variation of $f$ due to changes in the configurations of other molecules (which is represented by the term in $W$ ).

Concerning the changes in momentum, we may use the dilute gas assumption following the traditional interpretation, that is: account for the momentum changes of a molecule only during instantaneous collision with another molecule. Accordingly, the momentum changes that occur due to the internal forces in equation (10) can be approximated by a Boltzmann-type of collision integral. We can therefore write,

$$
\frac{\partial f}{\partial t}+(\xi \cdot \nabla) f+\left(F_{e x t} \cdot \nabla_{\xi}\right) f+W \frac{\partial f}{\partial v}=I(f, f) .
$$

In contrast to the dynamics of a single molecule, the microscopic parameter, $v$, is determined by the geometrical arrangement and dispersion of nearby molecules. These molecules include the nearest, the furthest away and even molecules that are not in the localized region. This variable does not impact on the localized microscopic dynamics of a single molecule but introduces a measure of the cohesive nature of the medium that has been previously ignored by the instantaneous collisional representation of the momentum exchanges.

Hereafter, we disregard body forces, i.e. $F_{\text {ext }}=0$ (situations with non-vanishing body force can in any case be easily incorporated into the description).

\subsection{A model for deriving macroscopic equations}

\subsubsection{Definition of macroscopic properties}

We define first the following average quantity:

$$
A_{n}(t, X)=\int_{-\infty}^{+\infty} \int_{0}^{+\infty} f(t, X, \xi, v) d_{v} d_{\xi}
$$

This quantity refers, according to the definition of the distribution function $f(t, X, \xi, v)$, to a reduced probability in the position space, i.e. the probability 
of finding a molecule around $X$ regardless of its velocity and the distribution of the other molecules. This is not therefore a proper thermodynamic massdensity of the medium.

The local mean value, $\bar{Q}(t, X)$, of any property $Q$ can be defined according to classical statistical mechanics by,

$$
\bar{Q}(t, X)=\frac{1}{A_{n}(t, X)} \int_{-\infty}^{+\infty} \int_{0}^{+\infty} Q f(t, X, \xi, v) d_{v} d_{\xi}
$$

For example, the local average of $v$, i.e. the local mean-free-volume around each gaseous molecule, is given by,

$$
\bar{v}(t, X)=\frac{1}{A_{n}(t, X)} \int_{-\infty}^{+\infty} \int_{0}^{+\infty} v f(t, X, \xi, v) d_{v} d_{\xi}
$$

From this mean value of the volume around a molecule we can define a massdensity in the vicinity of position $X$ through:

$$
\bar{\rho}(t, X)=\frac{A_{n}(t, X) M}{A_{n}(t, X) \bar{v}(t, X)}=\frac{M}{\bar{v}(t, X)},
$$

where $M$ is the molecular mass. The specific volume is then given by $\bar{v}(t, X) / M$.

Two mean velocities can be defined using two different weighting values. First, a local mean mass-velocity, $U_{m}(t, X)$, is given through

$$
A_{n}(t, X) U_{m}(t, X)=\iint \xi f(t, X, \xi, v) d_{\xi} d_{v}
$$

As the molecular mass is constant in single-component media, it has been canceled out in equation (16). According to the definition of the distribution function, this average velocity can be viewed as the average velocity at which molecules are traveling; it is independent of the mass-density of the medium.

Using the microscopic free volume as the weighting, a local mean volumevelocity, $U_{v}(t, X)$, can also be defined:

$$
\bar{v}(t, X) A_{n}(t, X) U_{v}(t, X)=\iint v \xi f(t, X, \xi, v) d_{\xi} d_{v} .
$$

If the spatial distributions of the molecules are such that molecules maintain on average the same separation distances between each other, in particular the measurable volume between the molecules is always and everywhere the same, then $v$ is a constant and it is seen that $U_{m}(t, X)$ and $U_{v}(t, X)$ coincide. This uniformity situation represents a homogeneous medium, where mass-density is constant throughout. It follows that a difference between these two velocities occurs in a non-homogeneous medium, where variations of mass-density exist. 
From these two local mean velocities, two peculiar velocities can also be introduced. The usual mass-velocity definition of peculiar velocity is

$$
C=\xi-U_{m}
$$

But another peculiar velocity may be given through the volume-velocity, i.e.

$$
C^{\prime}=\xi-U_{v} .
$$

\subsubsection{A set of macroscopic conservative equations}

Our starting microscopic model is equation (11). The right hand side of this equation is the Boltzmann collision integral for hard-sphere molecules. During collisions, mass, momentum and energy are conserved, according to classical dynamics. These three quantities are collision invariants in the description. Our new microscopic parameter, $v$, is not at first sight a collision invariant. However, strictly speaking, a collision in our modelling means a change only in the momentum of the molecules. The variable $v$ describes the remaining information about the microscopic system, in particular the changes in the arrangement of surrounding molecules. It is therefore not involved in the change of momentum of a target molecule, so we can consider this variable as invariant under the vanishing collision time.

We derive macroscopic equations in the following way. Consider a function $\psi \equiv \psi(\xi, v)$, that is, a microscopic property of a molecule represented as a function of the velocity and $v$. Then we take the integral of equation (11) over the velocity space and over the configuration space (i.e. over $v$ ) after multiplication by $\psi$ assuming, obviously, that the moments involved in these integrations exist. For conciseness, the detailed calculations are presented in Appendix A.

For $\psi=v$, the macroscopic equation obtained is,

$$
\frac{\partial A_{n} \bar{v}}{\partial t}+\nabla \cdot\left[A_{n} \bar{v} U_{m}\right]+\nabla \cdot\left[A_{n} \mathbf{J}_{v}\right]=A_{n} W
$$

where the quantity $\mathbf{J}_{v}$ denotes a flux of volume, defined with the velocity $C$ by

$$
\mathbf{J}_{v}=\frac{1}{A_{n}} \iint v C f d_{\xi} d_{v}
$$

For $\psi=1, \xi$ and $\xi^{2}$, we obtained, respectively,

$$
\begin{gathered}
\frac{\partial A_{n}}{\partial t}+\nabla \cdot\left[A_{n} U_{m}\right]=0 \\
\frac{\partial A_{n} U_{m}}{\partial t}+\nabla \cdot\left[A_{n} U_{m} U_{m}\right]+\nabla \cdot\left[A_{n} \mathbf{P}\right]=0
\end{gathered}
$$


and

$$
\begin{array}{r}
\frac{\partial}{\partial t}\left[\frac{1}{2} A_{n} U_{m}^{2}\right]+\frac{\partial}{\partial t}\left[A_{n} e_{i n}\right]+\nabla \cdot\left[\frac{1}{2} A_{n} U_{m}^{2} U_{m}+A_{n} e_{i n} U_{m}\right] \\
+\nabla \cdot\left[A_{n} \mathbf{P} \cdot U_{m}\right]+\nabla \cdot\left[A_{n} \mathbf{q}\right]=0,
\end{array}
$$

where the quantities $\mathbf{P}, e_{i n}$ and $\mathbf{q}$ denote:

$$
\begin{gathered}
\mathbf{P}_{i j}=\frac{1}{A_{n}} \iint C_{i} C_{j} f d_{\xi} d_{v}, \\
e_{i n}=\frac{1}{A_{n}} \iint \frac{1}{2} C^{2} f d_{\xi} d_{v}, \\
\mathbf{q}=\frac{1}{A_{n}} \iint \frac{1}{2} C^{2} C f d_{\xi} d_{v} .
\end{gathered}
$$

In total, we have a set of four macroscopic equations. The quantities $\mathbf{P}, e_{i n}$ and $\mathbf{q}$, which are all written using the peculiar velocity $C$ that is based on the mass-velocity $U_{m}$, are at this stage simply quantities appearing through the mathematical derivation of the macroscopic equations.

Equation (22) corresponds to the classical continuity equation. However, we note that this equation can be derived directly from equation (10) if the interaction potential is independent of the microscopic velocity of the molecules. In other words, this equation does not necessarily require Boltzmann collision modelling, with its assumption of conservation of mass during collisions. It cannot be said to be an equation describing the evolution of the mass-density of the medium as it involves only the average velocity of molecules and the reduced probability $A_{n}$. As mentioned earlier, two real interacting molecules change their positions during the interaction. Although conservation of mass evidently still holds in such a situation, this alone does not prescribe the spatial evolution of the mass-density of the medium in which the two molecules are interacting. The changes of position during interactions are required in a complete description of the evolution of the mass-density of the medium.

Furthermore, considering a set of $N$ arbitrary molecules, $N A_{n}$ may be regarded as a number density, and $N A_{n} d_{X}$ as a probable number of molecules around the position $X$ at time $t$. In this case, the above set of macroscopic equations remains the same, as only a certain multiplication factor appears in the distribution function. However, our new kinetic description departs from a statistically-independent system because the variable $v$ that describes the mass-density of the medium is in fact a correlation parameter. A first consequence of this is that our equations (20) and (22) distinguish between the variations of the mass-density of the medium and a vanishing divergence of the mass-velocity, the latter being interpreted in statistical mechanics as "incompressibility of the flow" (and not the fluid). 


\section{An unusual transport term}

Using the definitions of the mean velocities and peculiar velocities in equations (16) to (19), the following identities can be easily proved:

$$
\iint C^{\prime} f d_{\xi} d_{v}=-A_{n}\left(U_{v}-U_{m}\right), \quad \iint v C^{\prime} f d_{\xi} d_{v}=0
$$

and

$$
A_{n} \bar{v} U_{v}=\iint v U_{m} f d_{\xi} d_{v}+\iint v C f d_{\xi} d_{v}
$$

So equation (21) can be re-written:

$$
\mathbf{J}_{v}=\bar{v}\left(U_{v}-U_{m}\right)
$$

In equation (30), $\mathbf{J}_{v}$ characterizes a macroscopic motion defined by $\left(U_{v}-U_{m}\right)$, given that $U_{m}$ and $U_{v}$ are both macroscopic velocities by definition. The flux $\mathbf{J}_{v}$ therefore acts like a convective flux in which the convected element is the volume transported at the macroscopic velocity $\left(U_{v}-U_{m}\right)$, which will be nonzero if mass-density variations exist in the medium. Therefore, we attribute this velocity $\left(U_{v}-U_{m}\right)$ to macroscopic expansion and compression of the medium that the mean velocity $U_{m}$ does not encompass.

If $\delta V$ represents the macroscopic change of volume of a fluid element over time $\delta t$, then an approximate expansion/compression speed is given by, $\left\|\bar{v}^{-1} \mathbf{J}_{v}\right\| \approx$ $\delta\left(V^{1 / 3}\right) / \delta t$, where $\|$. $\|$ denotes the modulus of a vector. The velocity $\mathbf{J}_{v}$ is oriented in the direction from high density to low density in the case of an expansion, and vice versa for compression.

The macroscopic motion characterized by the flux $\mathbf{J}_{v}$ affects the classical interpretation of "convective fluxes". In our description, the term "convective flux" will include the macroscopic motion defined through the flux $\mathbf{J}_{v}$, as well as that motion traditionally described by the average velocity $U_{m}$. In other words, a diffusive flux, which is due to purely random molecular motions, is a flux in which neither the motion $U_{m}$ nor the macroscopic expansion/compression motion described by $\mathbf{J}_{v}$ exist. A diffusive flux of a property $\psi$ should therefore be written using:

$$
\iint\left[\xi-\frac{1}{\bar{v}} \mathbf{J}_{v}-U_{m}\right] \psi d_{v} d_{\xi}=\iint C^{\prime} \psi d_{v} d_{\xi}
$$

Now, let us consider the following expression,

$$
\mathbf{J}_{\bar{\rho}}=\frac{1}{A_{n}} \iint \bar{\rho} C^{\prime} f d_{v} d_{\xi}=\frac{1}{A_{n} \bar{v}} \iint M C^{\prime} f d_{v} d_{\xi} .
$$

According to the definition of diffusive fluxes, the flux $\mathbf{J}_{\bar{\rho}}$ corresponds to a diffusive flux of mass; strictly speaking, the element undergoing diffusion in this equation is the mass-density $\bar{\rho}$ from equation (15). 
Using the definitions of the mean velocities, equations (16) and (17), with the definition of the peculiar velocity $C^{\prime}$ in equation (19), it can be shown that

$$
\mathbf{J}_{\bar{\rho}}=-\bar{\rho}\left(U_{v}-U_{m}\right) \text {. }
$$

Combining equations (33) and (30), we obtain

$$
\frac{1}{\bar{\rho}} \mathbf{J}_{\bar{\rho}}=-\frac{1}{\bar{v}} \mathbf{J}_{v} .
$$

Equation (34) is a symmetrical relation between the flux of volume, $\mathbf{J}_{v}$, and the diffusive flux $\mathbf{J}_{\bar{\rho}}$.

As $\mathbf{J}_{\bar{\rho}}$ is a diffusive flux, according to equation (32), we apply the phenomenological Fick's law of diffusion, so that

$$
\mathbf{J}_{\bar{\rho}}=-\kappa_{m} \nabla \bar{\rho}
$$

where $\kappa_{m}$ is the mass diffusion coefficient (strictly speaking, the mass-density diffusion coefficient). From equation (34) we then obtain,

$$
\mathbf{J}_{v}=\frac{\kappa_{m} \bar{v}}{\bar{\rho}} \nabla \bar{\rho} .
$$

In summary, our unusual flux $\mathbf{J}_{v}$ embodies both a diffusive and a convective nature. We note that $\kappa_{m}$ has the dimension of (length) ${ }^{2} /$ time, which is the same dimension as kinematic viscosity.

\section{Diffusive fluxes of momentum and energy}

\subsection{The pressure tensor, heat flux and internal energy}

Diffusive fluxes are those from which all macroscopic motions, including macroscopic expansion/compression have been removed. These fluxes should therefore be associated with the velocity $C^{\prime}$ defined in equation (19), which may also be written,

$$
C^{\prime}=\xi-U_{m}-\frac{1}{\bar{v}} \mathbf{J}_{v} .
$$

Furthermore, equations (18), (19) and (30) yield

$$
C-C^{\prime}=U_{v}-U_{m}=\frac{1}{\bar{v}} \mathbf{J}_{v}
$$

We can then express the fluxes $\mathbf{q}$ and $\mathbf{P}_{i j}$, and the quantity $e_{i n}$ from equations (25) to (27), which were simply quantities defined using the peculiar velocity 
$C$, using the following decomposition:

$$
C=C^{\prime}+\left(C-C^{\prime}\right)=C^{\prime}+\left(U_{v}-U_{m}\right) .
$$

So $\mathbf{P}_{i j}(t, X)$ from equation (25) with equation (39) becomes

$$
\mathbf{P}_{i j}=\mathbf{P}_{i j}^{\prime}-\frac{1}{\bar{v}^{2}}\left(\mathbf{J}_{v} \mathbf{J}_{v}\right)_{i j},
$$

where $\mathbf{J}_{v} \mathbf{J}_{v} \equiv\left(\mathbf{J}_{v} \mathbf{J}_{v}\right)_{i j}$ is the second-order tensor obtained by the product of the coordinate components of vector $\mathbf{J}_{v}$, i.e. $\left(\mathbf{J}_{v} \mathbf{J}_{v}\right)_{i j}=\left(\mathbf{J}_{v}\right)_{i}\left(\mathbf{J}_{v}\right)_{j}$, and $\mathbf{P}^{\prime}$ is the diffusive momentum flux tensor per unit mass,

$$
\mathbf{P}^{\prime}{ }_{i j}(t, X)=\frac{1}{A_{n}} \iint C_{i}^{\prime} C_{j}^{\prime} f d_{v} d_{\xi} .
$$

A scalar pressure $p^{\prime}$ can then be introduced through the sum of the three diagonal terms of $\mathbf{P}^{\prime}{ }_{i j}$, using the unit volume of gas, i.e.

$$
3 p^{\prime}=\frac{M \mathbf{P}_{i i}^{\prime}}{\bar{v}} .
$$

From equations (26) and (39), $e_{i n}$ becomes

$$
e_{i n}=e_{i n}^{\prime}-\frac{1}{2 \bar{v}^{2}} \mathbf{J}_{v}^{2}
$$

where the actual internal energy $e_{\text {in }}^{\prime}$ per unit mass is given as

$$
e_{i n}^{\prime}=\frac{1}{A_{n}} \iint \frac{1}{2} C^{\prime 2} f d_{\xi} d_{v} .
$$

A temperature $T^{\prime}$ can also then be defined:

$$
\frac{3}{2} k T^{\prime}=M e_{i n}^{\prime} .
$$

Equations (42), (44) and (45) imply

$$
p^{\prime} \bar{v}=k T^{\prime} .
$$

However, it should be born in mind that equation (46), which resembles the ideal gas formula, follows on from the definitions of temperature and pressure, and these two variables both ultimately describe the internal energy. Therefore, the main quantity that should be considered is the internal energy $e_{i n}^{\prime}$, rather than systematically taking the gas to be perfect. For example, when considering an isothermal flow, equation (42) should be used while the equation for the internal energy will give an equation for the pressure irrespective of an ideal gas assumption. 
Using equation (39), the flux q, defined in equation (27), is re-written

$\mathbf{q}(t, X)=\frac{1}{A_{n}} \iint \frac{1}{2}\left[C^{\prime}+\left(U_{v}-U_{m}\right)\right]^{2} C^{\prime} f d_{\xi} d_{v}+\left(U_{v}-U_{m}\right) \frac{1}{A_{n}} \iint \frac{1}{2} C^{2} f d_{\xi} d_{v}$.

Then equations (28), (38) and (43) yield

$$
\mathbf{q}=\mathbf{q}^{\prime}+\frac{1}{\bar{v}} \mathbf{P}^{\prime} \cdot \mathbf{J}_{v}+\frac{1}{\bar{v}}\left(e_{i n}^{\prime}-\frac{1}{\bar{v}^{2}} \mathbf{J}_{v}^{2}\right) \mathbf{J}_{v},
$$

where the diffusive energy flux vector per unit mass, $\mathbf{q}^{\prime} \equiv \mathbf{q}^{\prime}(t, X)$, is given by

$$
\mathbf{q}^{\prime}=\frac{1}{A_{n}} \iint \frac{1}{2} C^{\prime 2} C^{\prime} f d_{\xi} d_{v} .
$$

Equation (43) describes the total internal energy $e_{i n}$ of a group of gaseous molecules as being composed of a "heat energy", $e_{i n}^{\prime}$, and an elastic energy component driven by $\mathbf{J}_{v}$. If we imagine this group of molecules moving from a high density region to a low density region, this will cause a dilatation of the group provided the quantity of mass, i.e. the number of molecules in the group, is not allowed to change. Molecules may move out of or into the group but the amount of mass should remain the same. According to the first law of thermodynamics, the heat energy must exclude any kind of potential energy. In equation (43) the term involving $\mathbf{J}_{v}$ is a potential energy because it is related to the density gradient or spatial distribution, and so represents the dilatation energy of the group. So the actual heat energy should correspond here to $e_{i n}^{\prime}$, not $e_{i n}$.

Let us consider a mechanical compression (or an expansion against a piston) of argon gas. The molecular mass of argon is $M=6.63 \times 10^{-26} \mathrm{~kg}$ and the Boltzmann constant is $k=1.38 \times 10^{-23} \mathrm{~J} / \mathrm{K}$. At a piston compression speed equivalent to $\bar{v}^{-1} \mathbf{J}_{v}=50 \mathrm{~m} / \mathrm{s}$, the difference in temperature due to the $\mathbf{J}_{v}$ term appearing in equation (43) is $4.0 \mathrm{~K}$. This is of the same order as a typical temperature variation during free expansion of a gas [26]. The differences produced by including $\mathbf{J}_{v}$ in the definitions of the thermodynamic parameters (temperature and pressure) will not be important in typical every-day gas flows, which is in agreement with the classical viewpoint about potential energies in the framework of the thermodynamics of near-equilibrium dilute gases. However, the impact of the diffusive flux $\mathbf{J}_{v}$, through the set of hydrodynamic equations, on highly non-equilibrium flows requires more attention.

\subsection{Constitutive models and the final set of hydrodynamic equations}

The hydrodynamic model in our new description is the four macroscopic equations (20), (22), (23) and (24), together with equations (40), (43), (45) and 
(48). The diffusive fluxes, according to the definition in equation (31), correspond to $\mathbf{P}^{\prime}$ for the momentum and $\mathbf{q}^{\prime}$ for the energy. To close our system, we can apply the phenomenological laws of diffusion to model these fluxes at first order. These are written similarly to the classical Newtonian law for the stress tensor within the pressure tensor, $\mathbf{P}^{\prime}$, and the analogous Fourier's law for the heat flux, $\mathbf{q}^{\prime}$, i.e.

$$
\begin{aligned}
\frac{M \mathbf{P}_{i j}^{\prime}}{\bar{v}} & =p^{\prime} \delta_{i j}-\mu^{\prime}\left(\frac{\partial U_{v_{i}}}{\partial X_{j}}+\frac{\partial U_{v_{j}}}{\partial X_{i}}\right)-\eta^{\prime} \frac{\partial U_{v_{k}}}{\partial X_{k}} \delta_{i j}, \\
\frac{M \mathbf{q}^{\prime}}{\bar{v}} & =-\kappa_{h}^{\prime} \nabla T^{\prime}, \\
\mathbf{J}_{v} & =\frac{\kappa_{m} \bar{v}}{\bar{\rho}} \nabla \bar{\rho},
\end{aligned}
$$

with $\mu^{\prime}$ a dynamic viscosity, $\kappa_{h}^{\prime}$ a heat conductivity, $\eta^{\prime}$ a bulk viscosity, $\kappa_{m}$ the mass diffusion coefficient, all to be determined in this new framework. Note that the macroscopic motion, from which the momentum flux $\mathbf{P}^{\prime}$ is defined as a diffusive flux, is $U_{v}=U_{m}+\bar{v}^{-1} \mathbf{J}_{v}$. Therefore the phenomenological law of diffusion used to express $\mathbf{P}^{\prime}$ is applied with a gradient taken over $U_{v}$, as written in equation (50). So momentum diffusion is not generated by the gradient of the mass-velocity $U_{m}$ only, but also can be generated by a gradient in the macroscopic expansion/compression $\left(U_{v}-U_{m}\right)$. By definition of the pressure equation (42), the tensor $M \mathbf{P}^{\prime}{ }_{i j} / \bar{v}-p^{\prime} \delta_{i j}$ is traceless; this implies $\frac{2}{3} \mu^{\prime}+\eta^{\prime}=0$. Also, in the expressions for the stress tensor and heat flux, the momentum flux density and the heat energy flux density are both based on the unit of real volume of the gas that is $\bar{v}$.

The complete set of new hydrodynamic equations is rewritten below, for convenience:

\section{Continuity}

$$
\frac{D A_{n}}{D t}=-A_{n} \nabla \cdot U_{m}
$$

\section{Mass-density}

$$
\frac{D \bar{\rho}}{D t}=\frac{\bar{\rho}^{2}}{M}\left[\frac{1}{A_{n}} \nabla \cdot\left[A_{n} \mathbf{J}_{v}\right]-W\right]
$$

\section{Momentum}

$$
A_{n} \frac{D U_{m}}{D t}=-\nabla \cdot A_{n}\left(\mathbf{P}^{\prime}-\frac{1}{\bar{v}^{2}} \mathbf{J}_{v} \mathbf{J}_{v}\right)
$$




\section{Energy}

$$
\begin{aligned}
A_{n} \frac{D}{D t}\left[\frac{1}{2} U_{m}^{2}+e_{i n}^{\prime}\right. & \left.-\frac{1}{2 \bar{v}^{2}} \mathbf{J}_{v}^{2}\right]= \\
& -\nabla \cdot A_{n}\left[\left(\mathbf{P}^{\prime}-\frac{1}{\bar{v}^{2}} \mathbf{J}_{v} \mathbf{J}_{v}\right) \cdot U_{m}\right] \\
& -\nabla \cdot A_{n}\left[\mathbf{q}^{\prime}+\frac{1}{\bar{v}} \mathbf{P}^{\prime} \cdot \mathbf{J}_{v}+\frac{1}{\bar{v}}\left(e_{i n}^{\prime}-\frac{1}{\bar{v}^{2}} \mathbf{J}_{v}^{2}\right) \mathbf{J}_{v}\right]
\end{aligned}
$$

where we denote the material derivative $D / D t \equiv \partial / \partial t+U_{m} \cdot \nabla$. (See Appendix $\mathrm{B}$ for variant expressions of these macroscopic and hydrodynamic equations.)

These equations are solved for five unknown fluid macroscopic parameters: the probability density $A_{n}$, the mass-density, $\bar{\rho}$, the mass-velocity, $U_{m}$, the pressure, $p^{\prime}$, and the internal energy, $e_{i n}^{\prime}$. The equation set is then closed by the constitutive model equations (50) to (52), $M e_{i n}^{\prime}=(3 / 2) k T^{\prime}$ or $p^{\prime}=(2 / 3) \bar{\rho} e_{i n}^{\prime}$, and $U_{v}=U_{m}+\bar{v}^{-1} \mathbf{J}_{v}$.

In the momentum equation (55), the macroscopic velocity on the left-hand-side is the mass-velocity, $U_{m}$, while on the right-hand-side, according to equation (50), appears the volume velocity, $U_{v}$. This modification to the momentum equation introduced by our new hydrodynamic description, when compared to the usual Navier-Stokes equation, recalls a recent modification proposed by Brenner [27]. That is to say, the replacement of the mass velocity appearing in the stress tensor by another velocity that depends on density gradient.

In the energy equation (56), in the heat flux and dissipation terms, appears a contribution due to work done by a variation of volume. Because, in this new kinetic model, volume variations refer to the domain occupied by a group of molecules, we interpret these complementary work terms as due to variations occurring through the boundary of the local domain occupied by a group of molecules.

\subsection{An expression for $W$}

In our microscopic modelling, $W=\delta v / \delta t$ appears as the rate of change of the free volumes between molecules. According to our description, this term manifests the cohesive nature of the molecules because the medium should not be described simply by the dynamics of a single molecule. Furthermore, the choice of the "volume" in our representation is not arbitrary. Volume is a configurational indication parameter in both continuum fluid mechanics and classical thermodynamics. In these cases, a change in $v$ is indicated by changes in a property of the ensemble. Because using Boltzmann modelling precludes an exact description of the field interactions between molecules, we 
assume that the rate of change of volume manifests through the variation of the thermodynamic properties, such as temperature and pressure (which are more likely to represent collective properties). We therefore write,

$$
W=\frac{\delta v}{\delta t}=\bar{v}\left(\frac{1}{\bar{v}} \frac{\partial v}{\partial T^{\prime}}\right)_{p^{\prime}} \frac{d T^{\prime}}{d t}+\bar{v}\left(\frac{1}{\bar{v}} \frac{\partial v}{\partial p^{\prime}}\right)_{T^{\prime}} \frac{d p^{\prime}}{d t}+\ldots
$$

or

$$
W=\bar{v} \alpha \frac{d T^{\prime}}{d t}-\bar{v} \chi \frac{d p^{\prime}}{d t}+\ldots,
$$

with compressibility coefficients defined by:

$$
\alpha=\left(\frac{1}{\bar{v}} \frac{\partial v}{\partial T^{\prime}}\right)_{p^{\prime}}, \quad \chi=-\left(\frac{1}{\bar{v}} \frac{\partial v}{\partial p^{\prime}}\right)_{T^{\prime}} .
$$

Using the above approximation for $W$, the mass-density equation (54) can be rewritten:

$$
\frac{D \bar{\rho}}{D t}=\frac{\bar{\rho}^{2}}{M A_{n}} \nabla \cdot\left[A_{n} \mathbf{J}_{v}\right]-\bar{\rho}\left(\alpha \frac{d T^{\prime}}{d t}-\chi \frac{d p^{\prime}}{d t}\right) .
$$

In this equation, the left-hand-side involves the material derivative based on $U_{m}$, while on the right-hand-side is a total derivative which is not systematically equal to this material derivative. We express the total variation at the macroscopic scale using the velocity $U_{v}$, rather than the mass velocity $U_{m}$, i.e

$$
\frac{d}{d t} \equiv \frac{\partial}{\partial t}+U_{m} \cdot \nabla+\frac{1}{\bar{v}} \mathbf{J}_{v} \cdot \nabla
$$

that is to say, following both the average mass motion and dilatation motion of the gas. We therefore have from equation (60)

$$
\frac{1}{\bar{\rho}} \frac{D \bar{\rho}}{D t}=\frac{\bar{\rho}}{M A_{n}} \nabla \cdot\left[A_{n} \mathbf{J}_{v}\right]-\alpha\left(\frac{D T^{\prime}}{D t}+\frac{1}{\bar{v}} \mathbf{J}_{v} \cdot \nabla T^{\prime}\right)+\chi\left(\frac{D p^{\prime}}{D t}+\frac{1}{\bar{v}} \mathbf{J}_{v} \cdot \nabla p^{\prime}\right) .
$$

By using equation (46) this equation may be re-written,

$$
\frac{\bar{\rho}}{M A_{n}} \nabla \cdot\left[A_{n} \mathbf{J}_{v}\right]+\frac{1}{\bar{v} \bar{\rho}} \mathbf{J}_{v} \cdot \nabla \bar{\rho}=\left(\frac{1}{p^{\prime}}-\chi\right) \frac{d p^{\prime}}{d t}-\left(\frac{1}{T^{\prime}}-\alpha\right) \frac{d T^{\prime}}{d t} .
$$

If we associate the microscopic to the macroscopic rate of change of $v$ (i.e. $d \bar{v} / d t=\delta v / \delta t$ ), according to equation (46) the coefficients $\alpha$ and $\chi$ may then be given, respectively, by $1 / T^{\prime}$ and $1 / p^{\prime}$. In this case, equation (63) reduces to

$$
\frac{\bar{\rho}}{M A_{n}} \nabla \cdot\left[A_{n} \mathbf{J}_{v}\right]+\frac{1}{\bar{v} \bar{\rho}} \mathbf{J}_{v} \cdot \nabla \bar{\rho}=0,
$$

which depends on the flow, i.e. $U_{m}$, via $A_{n}$. Equation (64) is a diffusion type of equation for the mass-density $\bar{\rho}$ if we eliminate the motion induced by $U_{m}$ 
in the flow by setting $A_{n}$ to be a constant. The advection field in this diffusion equation is given by $\mathbf{J}_{v}$, which confers a unique aspect to this diffusion equation and confirms again both the convective and diffusive characters of $\mathbf{J}_{v}$ discussed previously.

Finally, our new hydrodynamic equations (53) to (56) coincide with the NavierStokes set of equations when there are no mass-density variations, i.e. when $\mathbf{J}_{v}$ vanishes as well as $W$.

\section{Applications}

\subsection{Density profiles in a steady state heat transfer problem}

A first test case of our new hydrodynamic model is the prediction of massdensity profiles in rarefied gas flow in the continuum-transition regime. The test configuration is the heat conduction problem between two parallel plates at different temperatures, and is presented, for example, in reference [6]. There have been several previous investigations of this configuration because of the great interest in heat transfer descriptions and heat conductivity predictions in the continuum-transition regime, and because results from many previous theoretical models based on Boltzmann approximations provide hardly an acceptable result when compared with experiments.

Experimental data for the mass-density is determined by observing the luminescence produced by a high-energy electron beam traversed between the plates [6]. In [5] a finite-difference analysis of the nonlinear Boltzmann equation for hard-sphere molecules was used to solve the same flow configuration. According to [5], "there is a considerable difference between the mass-density distribution by the full Boltzmann equation and the experiments".

For this flow configuration, the classical set of Navier-Stokes equations reduce to the following: the continuity equation vanishes, the pressure is constant and the temperature profile is linear (from the heat conduction equation). So, strictly speaking, the three conventional hydrodynamic equations do not predict any actual mass-density profile.

We then consider our new hydrodynamic equations in a one-dimensional steady state configuration with $U_{m}=0$ (i.e. no gross mass motion). The $x$-axis will be normal to the plates. The continuity equation (53) vanishes and equation (64) gives,

$$
\bar{\rho} \frac{\partial}{\partial x}\left(\frac{\kappa_{m}}{\bar{\rho}^{2}} \frac{\partial \bar{\rho}}{\partial x}\right)+\kappa_{m}\left(\frac{1}{\bar{\rho}} \frac{\partial \bar{\rho}}{\partial x}\right)^{2}=0 .
$$


The experiment [6] was designed to avoid convection effects; in equation (65) the term involving the square of spatial derivatives of the mass-density came from the advection component appearing in equation (64), therefore the elimination of any convection effects allows us to neglect this term. Equation (65) then reduces to,

$$
\frac{\partial^{2} \bar{\rho}}{\partial x^{2}}=0
$$

which has the solution

$$
\bar{\rho}(x)=C_{t 2}+x C_{t 1} .
$$

Note that this mass-density profile is linear, and is obtained from the diffusion equation of the mass-density (not from an equation of state). According to experimental data [5], the mass-density profiles are clearly linear. Boundary conditions are required for the mass-density to specify the integration constants $C_{t 1}$ and $C_{t 2}$ if we are to provide a complete analysis using our model.

From equation (67), we can deduce the expression for the mean-free-volume around molecules, $\bar{v}$, through the relation $\bar{\rho}=M / \bar{v}$. As $U_{m}$ is zero, the profile for $U_{v}=\left(U_{v_{x}}, 0,0\right)$ is found using $(30)$ and (52), i.e.

$$
U_{v_{x}}(x)=\kappa_{m} \frac{C_{t 1}}{\bar{\rho}(x)}
$$

The momentum equation (55) with equations (50) and (52) gives,

$$
\frac{\partial}{\partial x}\left[\frac{p^{\prime}}{\bar{\rho}}-\frac{4}{3} \frac{\mu}{\bar{\rho}} \frac{\partial}{\partial x}\left(\frac{\kappa_{m}}{\bar{\rho}} \frac{\partial \bar{\rho}}{\partial x}\right)-\left(\frac{\kappa_{m}}{\bar{\rho}} \frac{\partial \bar{\rho}}{\partial x}\right)^{2}\right]=0 .
$$

Following the previous analysis that reduced the mass-density equation to equation (66), the momentum equation reduces to,

$$
\frac{\partial}{\partial x}\left(\frac{p^{\prime}}{\bar{\rho}}\right)=0 .
$$

Note at this stage that the perfect gas formula, equation (46), is a consequence of our definition of pressure $p^{\prime}$ and temperature $T^{\prime}$. That is to say, both temperature and pressure are defined from the same quantity, which is the internal energy, through equations (42) and (45). Therefore, the interpretation of equation (70) is that the pressure energy is constant and not that there is a constant temperature (as would follow from the perfect gas formula).

Furthermore, the full momentum equation (69) is written in terms of the velocity $U_{v}=\bar{v}^{-1} \mathbf{J}_{v}$, as

$$
\frac{\partial}{\partial x}\left[\frac{p^{\prime}}{\bar{\rho}}-\frac{4}{3} \frac{\mu}{\bar{\rho}} \frac{\partial U_{v_{x}}}{\partial x}-U_{v_{x}}^{2}\right]=0
$$


which shows that the full expression of the conservation equation of the pressure energy is

$$
\frac{p^{\prime}}{\bar{\rho}}-U_{v_{x}}^{2}-\frac{4}{3} \frac{\mu}{\bar{\rho}} \frac{\partial U_{v_{x}}}{\partial x}=C_{t p}
$$

and the full pressure profile, using equation (67), therefore

$$
p^{\prime}(x)=C_{t 2} C_{t p}+C_{t 1} C_{t p} x+\kappa_{m}^{2} \frac{C_{t 1}^{2}}{\bar{\rho}}-\frac{4}{3} \frac{\mu \kappa_{m}}{\bar{\rho}^{2}} C_{t 1}^{2},
$$

where here, and below, $C_{t \ldots}$ are integration constants.

The energy equation (56) reduces to,

$$
\frac{\partial}{\partial x}\left[\frac{\kappa_{h}^{\prime}}{\bar{\rho}} \frac{\partial T^{\prime}}{\partial x}-\frac{5}{2} U_{v_{x}} \frac{p^{\prime}}{\bar{\rho}}+\frac{4}{3} \frac{\mu}{\bar{\rho}} U_{v_{x}} \frac{\partial U_{v_{x}}}{\partial x}+U_{v_{x}}^{3}\right]=0,
$$

whence we can derive the temperature profile (using the mass-density and pressure profiles):

$$
T^{\prime}(x)=C_{t T 2}+\frac{C_{t T 1} C_{t 2}}{\kappa_{h}^{\prime}} x+\frac{5}{2} \frac{\kappa_{m}}{\kappa_{h}^{\prime}} C_{t 1} C_{t p} x+\frac{C_{t T 1} C_{t 1}}{2 \kappa_{h}^{\prime}} x^{2}-\frac{3}{2} \frac{\kappa_{m}^{3}}{\kappa_{h}^{\prime} \bar{\rho}} C_{t 1}^{2}+\frac{\mu \kappa_{m}^{2}}{\kappa_{h}^{\prime} \bar{\rho}^{2}} C_{t 1}^{2}
$$

Our new hydrodynamic model impacts on the pressure and temperature profiles mainly through the mass-density slope, $C_{t 1}$. In the approximation of an incompressible gas, where the mass-density could be viewed as constant, we have $C_{t 1}=0$; then equations (73) and (75) give, respectively, constant pressure and linear temperature profiles. These are the basic solutions known from the Navier-Stokes-Fourier set of equations.

More generally, however, our new model - even if we disregard any contributions from the volume diffusion terms (i.e. disregard any terms with coefficient $\left.\kappa_{m}\right)$ - predicts a linear pressure profile and a parabolic temperature profile, due to mass-density variations. The corrections introduced by our full model can be evaluated only if the temperature gradient throughout the domain is such that variations of mass-density affect the temperature profile: in other words, when the temperature difference between the two plates is large.

The classical hydrodynamic approach to correcting the profiles in the continuumtransition regime is to introduce a temperature-jump at the boundaries, with the restriction that $\Delta T^{\prime} / T^{\prime}$ is small [28]. However, in our new hydrodynamic model the profile correction results from important variations of thermodynamic properties. Moreover, $\Delta T^{\prime} / T^{\prime}$ is not always restricted to small values and the correction to the temperature distribution is not always due to boundary effects, as is also shown in the following example. 


\subsection{Shock waves and large temperature jumps}

Consider a shock wave in a rarefied gas flow, within which a rapid variation of temperature (at constant pressure) exists over a distance of the order of a molecular mean free path, $\lambda_{m}$, which is of the same order of magnitude as the system characteristic length defined by the shock density gradient. In this case, we approximate the expansion/compression by $\left\|\bar{v}^{-1} \mathbf{J}_{v}\right\| \approx \lambda_{m} / \tau_{v}$ where $\tau_{v}$ is the characteristic time of diffusion of volume (or mass-density). Accordingly, the temperature difference induced by the diffusive component $\mathbf{J}_{v}$ in equation (43) is,

$$
\Delta T^{\prime} \approx \frac{M}{3 k}\left(\frac{\lambda_{m}}{\tau_{v}}\right)^{2} .
$$

If we hypothesise that the diffusion time $\tau_{v}$ is similar to the diffusion time for energy, then the ratio $\lambda_{m} / \tau_{v}$ is close to the thermal speed and we have $\left(\lambda_{m} / \tau_{v}\right)^{2} \approx 3 k T^{\prime} / M$. Consequently, the temperature difference $\Delta T^{\prime} \approx T^{\prime}$, i.e. the same order of magnitude as the kinetic temperature $T^{\prime}$ (the temperature commonly identified with the flow).

This temperature difference is similar in magnitude to those observed in high speed rarefied gas flows during space vehicle re-entry, for which the classical theory of surface temperature jump is known to have serious shortcomings [29].

\section{Conclusions}

We have discussed the mathematical representation of a dilute gas, and introduced a modified kinetic description that includes a supplementary spatial configuration parameter. In doing this, we relaxed some aspects of the modelling of spatial configurations of molecules that are dictated by the standard description of the Boltzmann kinetic equation. The set of macroscopic conservation equations derived from our modified kinetic approach comprises four equations rather than the usual three; an evolution equation purely of the mass-density is added to the set of three conservation equations, while the classical continuity equation is replaced by a conservation equation for the reduced probability density. The equation for the mass-density turns out to be a mass-diffusion equation. We therefore distinguish between conservation of mass and the variation of the mass-density as a thermodynamic parameter to account for any variation in the volume occupied by the medium.

An unconventional form of transport, having both convective and diffusive characteristics, appeared, which led us to re-classify the diffusive and convective fluxes. As a result, we obtained a hydrodynamic model in which contri- 
butions due to mass-density variations appear in the momentum and energy equations.

Our new model should show most departure from conventional fluid dynamic descriptions in micro flow cases, gas flows involving sensitive mass-density variations (such as shock waves), and highly compressible rarefied gas flows. Some qualitative comparisons between the new description and experimental observations have been presented above; future work will include a more complete quantitative analysis, in addition to the investigation of boundary conditions.

\section{Acknowledgements}

This work is funded in the UK by the Engineering and Physical Sciences Research Council under grant EP/D007488/1, and through a Philip Leverhulme Prize for JMR from the Leverhulme Trust. The authors would like to thank Howard Brenner of MIT (USA), Gilbert Méolans of the Université de Provence (France), and Chris Greenshields of Strathclyde University (UK) for useful discussions, as well as the referees of this paper for their helpful comments. JMR would also like to thank the President and Fellows of Wolfson College, Cambridge, and John Young of the Engineering Department, Cambridge University, for their hospitality during a sabbatical year when, through their stimulating support, this work started to take shape.

\section{References}

[1] H. Struchtrup, Macroscopic Transport Equations for Rarefied Gas Flows: Approximation Methods in Kinetic Theory, Springer, 2005.

[2] C. Cercignani, R. Illner, M. Pulvirenti, Theory and Application of the Boltzmann Equation, Springer Heidelberg, 1994.

[3] P. Lipavsky, K. Morawetz, V. Spicka, Kinetic equation for strongly interacting dense Fermi systems, Vol. 26, EDP sciences, Annales de Physique, 2001.

[4] S. Chapman, T. Cowling, The Mathematical Theory of Non-Uniform Gases, 3rd Edition, Cambridge Mathematical Library, 1970.

[5] T. Ohwada, Heat flow and temperature and density distributions in a rarefied gas between parallel plates with different temperatures: finite-difference analysis of the nonlinear Boltzmann equation for hard-sphere molecules, Physics of Fluids 8 (8) (1996) 2153-2160. 
[6] W. P. Teagan, G. S. Springer, Heat transfer and density-distribution measurements between parallel plates in the transition regime, Physics of Fluids 11 (3) (1968) 497-506.

[7] E. P. Gross, E. A. Jackson, Kinetic models and the linearized Boltzmann equation, Physics of Fluids 2 (4) (1959) 432-441.

[8] Y. V. N. Victor Yu. Alexandrov, Oscar G. Friedlander, Numerical and experimental investigations of thermal stress effect on nonlinear thermomolecular pressure difference, in: 23rd International Symposium on Rarefied Gas Dynamics, Vol. 663, 2002, pp. 250-257.

[9] V. S. Galkin, M. N. Kogan, Derivation of the equations of slow nonisothermal gas flows, Fluid Dynamics 14 (6) (1979) 77-84.

[10] J. R. Bielenberg, H. Brenner, A continuum model of thermal transpiration, Journal of Fluid Mechanics 456 (2006) 1-23.

[11] E. G. D. Cohen, Kinetic theory of non-equilibrium fluids, Physica A 118 (1983) $17-42$.

[12] R. K. K. Agarwal, K.-Y. Yun, R. Balakrishnan, Beyond Navier-Stokes: Burnett equations for flows in the continuum-transition regime, Physics of Fluids 13 (10) (2001) 3061-3085.

[13] L. C. Woods, Frame-indifferent kinetic theory, Journal of Fluid Mechanics 136 (1983) 423-433.

[14] P. J. Dellar, Macroscopic descriptions of rarefied gases from the elimination of fast variables, Physics of Fluids 19 (10) (2007) 107101.

[15] J. M. Reese, M. A. Gallis, D. A. Lockerby, New directions in fluid dynamics: nonequilibrium aerodynamic and microsystem flows, Philosophical Transactions of the Royal Society of London A 361 (2003) 2967-2988.

[16] S. K. Dadzie, J. M. Reese, C. R. McInnes, A volume-based description of gas flows with localised mass-density variations, arXiv:physics/0611206v1.

[17] C. Cercignani, Rarefied Gas Dynamics: From Basic Concepts to Actual Calculations, Cambridge University Press, 1990.

[18] C. Cercignani, Theory and Application of the Boltzmann Equation, Scottish Academic Press, 1975.

[19] B. Leaf, Derivation of the Boltzmann equation from the Liouville equation, Physica 59 (2) (1972) 206-227.

[20] P. S. Lee, T.-Y. Wu, Boltzmann equation with fluctuations, International Journal of Theoretical Physics 7 (4) (1973) 267-276.

[21] J. M. Blatt, A. H. Opie, A new derivation of the Boltzmann transport equation, Journal of Physics A 7 (9) (1974) 1895-1906. 
[22] P. Degond, L. Pareschi, G. Russo, Modeling and Computational Methods for Kinetic Equations, Birkhäuser, 2004.

[23] J. C. Maxwell, On stresses in rarified gases arising from inequalities of temperature, Philosophical Transactions of the Royal Society of London 170 (1878) 231-256.

[24] J. S. Rowlinson, The Maxwell-Boltzmann distribution, Molecular Physics $103(21-23)(2005) 2821$.

[25] C. Callender, A collision between dynamics and thermodynamics, Entropy 6 (2004) 11-20.

[26] E. H. Kennard, Kinetic Theory of Gases, McGraw-Hill Book Company Inc, 1938.

[27] H. Brenner, Navier-Stokes revisited, Physica A 349 (1-2) (2005) 60-132.

[28] P. Welander, On the temperature jump in rarefied gas, Arkiv für Fysik 7 (44) (1954) 507.

[29] R. N. Gupta, C. D. Scott, Comment on corrective term in wall slip equations for Knudsen layer, AIAA Journal of Thermophysics and Heat Transfer 10 (1) (1996) 190-191.

\section{A Conservation equations from the modified kinetic equation}

We present here the derivation of macroscopic equations from our kinetic equation (11). This procedure is similar to the classical one: equation (11) is multiplied by the microscopic quantities $v, M,(M \xi),\left(M \xi^{2}\right)$ and then the result is integrated over $d_{v}$ and $d_{\xi}$. In this process it should be kept in mind that $t, X, \xi$, and $v$ are independent variables, while any mean value of a microscopic quantity given through definition (13) depends on $t$ and $X$.

- Conservation of volume. Multiplying equation (11) by the microscopic element $v$, and integrating over $v$ and $\xi$, we obtain,

$$
\iint v \frac{\partial f}{\partial t} d_{v} d_{\xi}+\iint v(\xi \cdot \nabla) f d_{v} d_{\xi}+W \iint v \frac{\partial f}{\partial v} d_{v} d_{\xi}=0
$$

where the collision integral term vanishes. Since $t, X, \xi$ and $v$ are independent variables, this equation reduces to

$$
\iint \frac{\partial v f}{\partial t} d_{v} d_{\xi}+\iint \nabla \cdot(v f \xi) d_{v} d_{\xi}+W \iint v \frac{\partial f}{\partial v} d_{v} d_{\xi}=0
$$


which can also be written,

$$
\frac{\partial A_{n} \bar{v}}{\partial t}+\iint \nabla \cdot\left(v f U_{m}\right) d_{v} d_{\xi}+\iint \nabla \cdot(v f C) d_{v} d_{\xi}+W \iint v \frac{\partial f}{\partial v} d_{v} d_{\xi}=0 .
$$

Using partial integration and the integrability condition, $\lim _{v \rightarrow+\infty}(v f)=0$, the third integral term in relation (A.3) gives

$$
W \iint v \frac{\partial f}{\partial v} d_{v} d_{\xi}=-A_{n} W
$$

and relation (A.3) can then be written

$$
\frac{\partial A_{n} \bar{v}}{\partial t}+\nabla \cdot\left[A_{n} \bar{v} U_{m}\right]+\nabla \cdot\left[\iint(v C f) d_{v} d_{\xi}\right]-A_{n} W=0 .
$$

Finally, if we denote

$$
\mathbf{J}_{v}=\frac{1}{A_{n}} \iint(v C f) d_{v} d_{\xi}
$$

then the first macroscopic equation obtained is an evolution equation for the volume, and is written

$$
\frac{\partial A_{n} \bar{v}}{\partial t}+\nabla \cdot\left[A_{n} \bar{v} U_{m}\right]+\nabla \cdot\left[A_{n} \mathbf{J}_{v}\right]=A_{n} W
$$

- Conservation of mass. Multiplying equation (11) by the molecular mass $M$, and integrating over $v$ and $\xi$, we obtain:

$$
\iint M \frac{\partial f}{\partial t} d_{v} d_{\xi}+\iint M(\xi \cdot \nabla) f d_{v} d_{\xi}+W \iint M \frac{\partial f}{\partial v} d_{v} d_{\xi}=0
$$

where the collision integral term vanishes. The third integral term in equation (A.8) is zero owing to the generalized function character of $f$, i.e. $\lim _{v \rightarrow 0} f=0$ and $\lim _{v \rightarrow+\infty} f=0$. The second macroscopic equation obtained in this case is then:

$$
\frac{\partial M A_{n}}{\partial t}+\nabla \cdot\left[M A_{n} U_{m}\right]=0
$$

Combining equation (A.9) with the volume equation (A.7) gives

$$
\frac{\partial \bar{v}}{\partial t}+U_{m} \cdot \nabla \bar{v}+\frac{1}{A_{n}} \nabla \cdot\left[A_{n} \mathbf{J}_{v}\right]=W .
$$

Using the density $\bar{\rho}=M / \bar{v}$, this can be rewritten:

$$
\left(\frac{\partial \bar{\rho}}{\partial t}+U_{m} \cdot \nabla \bar{\rho}\right)-\frac{\bar{\rho}^{2}}{M A_{n}} \nabla \cdot\left[A_{n} \mathbf{J}_{v}\right]+\frac{\bar{\rho}^{2}}{M} W=0 .
$$


- Conservation of momentum. Multiplying equation (11) by $M \xi$, and integrating over $v$ and $\xi$, we obtain

$$
\iint M \xi \frac{\partial f}{\partial t} d_{v} d_{\xi}+\iint M \xi(\xi \cdot \nabla) f d_{v} d_{\xi}+W \iint M \xi \frac{\partial f}{\partial v} d_{v} d_{\xi}=0
$$

where the collision integral term vanishes. As $t$ and $\xi$ are independent variables, this equation can be written in the form:

$$
\iint \frac{\partial M f \xi}{\partial t} d_{v} d_{\xi}+\iint \nabla \cdot\left(M f \xi_{i} \xi_{j}\right) d_{v} d_{\xi}+W \iint M \xi \frac{\partial f}{\partial v} d_{v} d_{\xi}=0
$$

where $\xi_{i} \xi_{j}$ is the second order tensor constituted by the elements $\left(\xi_{i} \xi_{j}\right)$. Here also, the third integral term in relation (A.13) is zero owing to the generalized function character of $f$, i.e. $\lim _{v \rightarrow 0} \xi f=0$ and $\lim _{v \rightarrow+\infty} \xi f=0$. Then, using the definition of the peculiar velocity $C$, we obtain the third conservation equation:

$$
\frac{\partial M A_{n} U_{m}}{\partial t}+\nabla \cdot\left[M A_{n} U_{m} U_{m}\right]+\nabla \cdot\left[M A_{n} \mathbf{P}\right]=0
$$

where we denote $\mathbf{P} \equiv \mathbf{P}_{i j}(t, X)$ the flux:

$$
\mathbf{P}_{i j}(t, X)=\frac{1}{A_{n}} \iint\left(C_{i} C_{j}\right) f d_{v} d_{\xi}
$$

Using the mass conservation equation (A.9), the momentum equation may be written :

$$
A_{n}\left(\frac{\partial U_{m}}{\partial t}+U_{m} \cdot \nabla U_{m}\right)+\nabla \cdot\left[A_{n} \mathbf{P}\right]=0
$$

- Conservation of energy. Multiplying equation (11) by $\frac{1}{2} M \xi^{2}$ and integrating over $v$ and $\xi$, we obtain

$$
\iint \frac{1}{2} M \xi^{2} \frac{\partial f}{\partial t} d_{v} d_{\xi}+\iint \frac{1}{2} M \xi^{2}(\xi \cdot \nabla) f d_{v} d_{\xi}+W \iint M \xi^{2} \frac{\partial f}{\partial v} d_{v} d_{\xi}=0
$$

which, following the independent variable properties, becomes

$$
\iint \frac{1}{2} M \frac{\partial f \xi^{2}}{\partial t} d_{v} d_{\xi}+\iint \frac{1}{2} M \nabla \cdot\left(\xi^{2} f \xi\right) d_{v} d_{\xi}+W \iint M \xi^{2} \frac{\partial f}{\partial v} d_{v} d_{\xi}=0
$$

Here also, owing to the properties of $f$, i.e. $\lim _{v \rightarrow 0} \xi^{2} f=0$ and $\lim _{v \rightarrow+\infty} \xi^{2} f=$ 0 , the third integral term vanishes. Using the definitions of the velocities $U_{m}$ and $C$, we therefore have

$$
\begin{array}{r}
\frac{\partial}{\partial t}\left[\frac{1}{2} M A_{n} U_{m}^{2}\right]+\frac{\partial}{\partial t}\left[M A_{n} e_{i n}\right]+\nabla \cdot\left[\frac{1}{2} M A_{n} U_{m}^{2} U_{m}+M A_{n} e_{i n} U_{m}\right] \\
+\nabla \cdot\left[M A_{n} \mathbf{P} \cdot U_{m}\right]+\nabla \cdot\left[M A_{n} \mathbf{q}\right]=0,
\end{array}
$$


where we have introduced the quantity $e_{i n}$ that is given through:

$$
e_{i n}(t, X)=\frac{1}{A_{n}} \iint \frac{1}{2} C^{2} f d_{\xi} d_{v}
$$

and the flux $\mathbf{q}$, given by:

$$
\mathbf{q}(t, X)=\frac{1}{A_{n}} \iint \frac{1}{2} C^{2} C f d_{\xi} d_{v} .
$$

Equation (A.19) is the mean energy evolution equation. By using the mass conservation equation (A.9), the energy equation may be rewritten:

$$
\begin{aligned}
A_{n} \frac{\partial}{\partial t}\left[\frac{1}{2} U_{m}^{2}+e_{i n}\right] & +A_{n} U_{m} \cdot \nabla\left[\frac{1}{2} U_{m}^{2}+e_{i n}\right] \\
& +\nabla \cdot\left[A_{n} \mathbf{P} \cdot U_{m}\right]+\nabla \cdot\left[A_{n} \mathbf{q}\right]=0
\end{aligned}
$$

\section{B Other forms of the new hydrodynamic equations}

Multiplying the momentum equation (55) scalarly by $U_{m}$, we obtain

$$
A_{n} U_{m} \cdot \frac{D U_{m}}{D t}=-U_{m} \cdot\left[\nabla \cdot A_{n}\left(\mathbf{P}^{\prime}-\frac{1}{\bar{v}^{2}} \mathbf{J}_{v} \mathbf{J}_{v}\right)\right]
$$

which can be rewritten,

$$
A_{n} \frac{1}{2} \frac{D U_{m}^{2}}{D t}=-\nabla \cdot\left[A_{n}\left(\mathbf{P}^{\prime}-\frac{1}{\bar{v}^{2}} \mathbf{J}_{v} \mathbf{J}_{v}\right) \cdot U_{m}\right]+A_{n}\left(\mathbf{P}^{\prime}-\frac{1}{\bar{v}^{2}} \mathbf{J}_{v} \mathbf{J}_{v}\right): \nabla U_{m}
$$

Therefore, the energy equation (56) can also be re-written by using this expression of the momentum equation as,

$$
\begin{aligned}
& A_{n} \frac{D}{D t}\left[e_{i n}^{\prime}-\frac{1}{2 \bar{v}^{2}} \mathbf{J}_{v}^{2}\right]+\nabla \cdot A_{n}\left[\left(e_{i n}^{\prime}-\frac{1}{\bar{v}^{2}} \mathbf{J}_{v}^{2}\right) \frac{1}{\bar{v}} \mathbf{J}_{v}\right]= \\
& -\nabla \cdot A_{n}\left[\mathbf{q}^{\prime}+\mathbf{P}^{\prime} \cdot \frac{1}{\bar{v}} \mathbf{J}_{v}\right]-A_{n}\left(\mathbf{P}^{\prime}-\frac{1}{\bar{v}^{2}} \mathbf{J}_{v} \mathbf{J}_{v}\right): \nabla U_{m}
\end{aligned}
$$

